\title{
Large Colon
}

National Cancer Institute

\section{Source}

National Cancer Institute. Large Colon. NCI Thesaurus. Code C92439.

The ascending colon of the horse. 